\title{
Prostatic Ductal Adenocarcinoma: A Mini Review
}

\author{
Jonathan I. Epstein \\ Departments of Pathology, Urology, and Oncology, The Johns Hopkins Hospital, Baltimore, Md., USA
}

\author{
Key Words \\ Prostate adenocarcinoma - Ductal adenocarcinoma . \\ Gleason grade
}

\begin{abstract}
Prostatic ductal adenocarcinomas may arise either in large primary periurethral prostatic ducts or in the peripheral prostatic ducts. Ductal adenocarcinomas are composed of tall columnar cells arranged in cribriform, papillary, solid, single glands, and PIN-like patterns. Other than the prostatic intraepithelial neoplasia (PIN)-like ductal pattern, which behaves like Gleason pattern 3, ductal adenocarcinoma is comparable to Gleason pattern 4 prostate cancer. Ductal adenocarcinoma can have a patchy basal cell layer and typically expresses prostate-specific antigen (PSA) immunohistochemically. Mimickers of ductal adenocarcinoma include prostatic urethral polyps, hyperplastic benign prostate glands, high-grade PIN, colorectal adenocarcinoma, and papillary urothelial carcinoma. Copyright $\odot 2009 \mathrm{~S}$. Karger AG, Basel
\end{abstract}

Prostatic duct adenocarcinomas arising in large primary periurethral prostatic ducts often are associated with a normal digital rectal examination. Patients typically present with either urinary obstructive symptoms or gross/microscopic hematuria, and are diagnosed on transurethral resection. These tumors grow as exophytic lesions into the urethra, most commonly in and around the verumontanum. Tumors arising in the more peripheral prostatic ducts present as usual acinar prostate adenocarcinoma and are diagnosed on needle biopsy. Ductal adenocarcinomas are associated with variable serum prostate-specific antigen (PSA) levels [1].

While most adenocarcinomas of the prostate are composed of cuboidal cells arranged in acini, $0.4-0.8 \%$ of prostate cancers show a pure tumor composed of distinctive tall columnar cells of prostatic duct adenocarcinoma [2-5]. Prostatic duct adenocarcinomas show a variety of architectural patterns, often admixed in a single tumor. The two most common patterns are papillary and cribriform. The formation of papillary fronds is a distinctive architectural pattern not seen with usual acinar adenocarcinoma of the prostate. The cribriform pattern of prostatic duct adenocarcinoma is somewhat reminiscent of endometrial adenocarcinoma within the female, and is one of the reasons why these tumors were initially described as 'endometrioid carcinomas of the prostate' [6]. This pattern of prostatic adenocarcinoma differs from the cribriform pattern of prostatic acinar adenocarcinoma, which is composed of cuboidal epithelium and punched-out round lumina. Prostatic duct adenocarcinomas may also grow as solid nests or cribriform glands

\section{KARGER \\ Fax +4161306 1234 \\ E-Mail karger@karger.ch}

www.karger.com
(C) 2009 S. Karger AG, Basel

1011-7571/10/0191-0082\$26.00/0

Accessible online at:

www.karger.com/mpp
Prof. Jonathan I. Epstein

Departments of Pathology, Urology, and Oncology, The Johns Hopkins Hospital 600 N. Wolfe Street

Baltimore, MD 21287 (USA)

Tel. +1 410955 5043, Fax +1 410955 0115, E-Mail jepstein@jhmi.edu 
with central necrosis; this pattern cannot be distinguished from poorly differentiated prostatic acinar adenocarcinoma without seeing the solid pattern in association with papillary or cribriform prostatic duct adenocarcinoma. Single glands lined by tall columnar epithelial cells resembling adenocarcinoma of the colon represent another relatively uncommon pattern of prostatic ductal adenocarcinoma. A more recently described morphological variant of ductal adenocarcinoma is composed of simple glands lined by stratified columnar epithelium with cytological and architectural features of flat and tufting high-grade prostatic intraepithelial neoplasia, referred to as 'PIN-like ductal adenocarcinoma' $[7,8]$.

Ductal adenocarcinomas may show a range of cytological atypia ranging from very bland columnar cells to those with overt malignant features. Most cases of prostatic duct adenocarcinoma have amphophilic cytoplasm, although occasional cases with predominantly clear cells exist. In contrast to acinar carcinoma, ductal adenocarcinoma may be accompanied by a prominent stromal desmoplastic reaction, sometimes with hemosiderin deposition. While prostatic duct adenocarcinoma can be the sole component, more frequently it is found admixed with tumor showing acinar differentiation. In most cases with mixed acinar and ductal features, the two components are intimately comingled. One can also see a centrally located duct carcinoma with a peripherally located acinar tumor. Mixed ductal and acinar adenocarcinoma is encountered in about $5 \%$ of prostatic carcinoma cases.

Ductal adenocarcinomas express PSA and prostaticspecific acid phosphatase (PSAP), verifying their prostatic origin. Ductal adenocarcinomas, as they arise in ducts, may show residual staining for high molecular weight cytokeratin. Basal cells are detectable by p63 and HMWCK in a patchy fashion in about one third of ductal adenocarcinomas, which is the same as is seen in cribriform pattern 4 acinar adenocarcinomas [9]. Cases where biopsy shows all of the ductal adenocarcinoma within ducts outlined by basal cell staining should be treated the same as ductal adenocarcinoma identified without a basal cell layer. Seventy-seven percent of ductal prostatic adenocarcinomas show positive staining for AMACR, which is similar to the expression seen in Gleason pattern 4 acinar adenocarcinoma [9].

Ductal adenocarcinoma can be mistaken for a wide spectrum of benign, precancerous, and malignant entities. One of the lesions most frequently confused with cytologically bland ductal adenocarcinoma is prostatic urethral polyp. Whereas ductal adenocarcinomas are composed of tall pseudostratified columnar cells, prostatic urethral polyps are polypoid nodules made up of entirely benign-appearing prostate acini lined by prostatic glandular epithelium and urothelium. Ductal adenocarcinoma on needle biopsy may be particularly difficult to recognize as malignant, and can be underdiagnosed as 'hyperplastic' benign glands as there may be mild cytological atypia without prominent nucleoli [1]. The other feature that can result in underdiagnosis of prostatic duct adenocarcinoma on needle biopsy is tumor fragmentation, resulting in small detached foci of carcinoma.

A difficult distinction lies between cribriform highgrade PIN and ductal adenocarcinoma of the prostate. Ductal adenocarcinomas often contain true papillary fronds with well-established fibrovascular cores, whereas high-grade PIN more frequently reveals micropapillary fronds with tall columns of epithelium without fibrovascular stalks. Ductal adenocarcinomas frequently contain comedonecrosis, which may be extensive. Highgrade PIN typically lacks comedonecrosis, and when necrosis is present, it is focal. Finally, ductal adenocarcinomas may consist of very large and/or back-to-backglands, whereas glands involved in PIN are of the size and distribution of benign glands. The use of basal cell markers in this differential diagnosis may be problematical, as both high-grade PIN and ductal adenocarcinoma may display a patchy basal cell layer. However, absence of a basal cell layer in numerous glands rules out PIN [10]. PIN-like ductal adenocarcinoma is even more difficult to differentiate from high-grade PIN [7]. Ductal adenocarcinoma can be diagnosed either because the atypical glands are too crowded to represent high-grade PIN or there are too many atypical glands that are negative for basal cell markers to be consistent with high-grade PIN. In some needle biopsy specimens, there are only a few atypical glands with papillary fronds that are negative for basal cell markers that are highly suspicious for ductal adenocarcinoma, yet due to the limited number of atypical glands, high-grade PIN can not be ruled out with certainty. These cases can be reported as: 'Atypical glands where the differential diagnosis is between highgrade PIN and ductal adenocarcinoma. Repeat biopsy is recommended.'

Ductal adenocarcinoma, especially the pattern characterized by single non-cribriform glands, can be difficult to distinguish from colorectal adenocarcinoma invading the prostate. Immunohistochemical demonstration of PSA and PSAP in ductal adenocarcinoma can verify the diagnosis. The finding of more characteristic 
patterns of prostatic ductal adenocarcinoma, such as the papillary pattern, or identifying admixed usual acinar adenocarcinoma can also aid in ruling out colonic adenocarcinoma. Only rarely are ductal adenocarcinomas associated with either extracellular or intracellular mucin. Negative immunohistochemical staining for B-catenin, CDX-2, and villin can be of further utility in excluding colon cancer $[7,11]$.

Prostatic duct adenocarcinoma on transurethral resection specimens can also mimic papillary urothelial carcinoma. The nuclei in urothelial carcinoma tend to be more pleomorphic, angulated in shape, contain variably numbered and sized nucleoli, and lack the columnar appearance of ductal adenocarcinoma [12]. Immunohistochemical demonstration of PSA and PSAP positivity and negative thrombomodulin and uroplakin staining in prostatic duct adenocarcinoma can confirm the diagnosis in difficult cases [13-15].

Prostatic ductal adenocarcinoma generally spreads in the same manner and should be treated in an analogous fashion to usual acinar adenocarcinoma of the prostate. There is, however, a greater propensity to spread to the testis and penis [16]. Ductal adenocarcinoma metastatic to the lung may be misdiagnosed as a primary lung tumor [17].

A unique aspect of this entity is that the urologist takes only a limited transurethral biopsy of the prostate, with the entire specimen consisting of only a small focus of prostatic duct adenocarcinoma. These tumor foci represent the 'tip of the iceberg', where there is more extensive unsampled carcinoma involving the underlying ductal system. Despite the limited tumor on biopsy or visible at cystoscopy, these tumors should be considered as aggressive lesions and treated accordingly. The one exception to considering ductal adenocarcinoma as aggressive tumors is the rare case when there is good sampling of the pros- tate with a sizable transurethral resection, and there is only a small focus of ductal adenocarcinoma; the prognosis in this situation is unknown.

Most studies have demonstrated that ductal morphology connotes a more aggressive course than acinar prostate cancer [18]. Men with ductal adenocarcinoma on needle biopsy who subsequently undergo radical prostatectomy have larger tumors with more advanced pathologic stage compared to acinar carcinoma and a shortened time to progression. Most ductal adenocarcinomas should be graded as Gleason score $4+4=8$, while retaining the diagnostic term of ductal adenocarcinoma to denote their unique clinical and pathological findings [19]. This can be achieved by diagnosing such a tumor as 'prostatic ductal adenocarcinoma (Gleason score $4+4=8$ )'. In cases with mixed ductal and acinar patterns, the ductal patterns should be assigned Gleason pattern 4 . For PINlike ductal adenocarcinomas, the evidence indicates that they behave comparable to Gleason score $3+3=6$. The finding of comedonecrosis warrants assignment of Gleason pattern 5 .

\section{Summary}

Ductal adenocarcinoma of the prostate is an unusual variant of prostate adenocarcinoma that has unique morphological and in some cases clinical features. It is critical to distinguish ductal adenocarcinoma from a variant of benign, premalignant, and malignant mimickers. Although typically ductal adenocarcinomas of the prostate are associated with a poor prognosis, there are several situations (i.e. limited cancer on an extensive transurethral resection, PIN-like ductal adenocarcinoma) in which the prognosis may not be as adverse.

\section{References}

1 Brinker DA, Potter SR, Epstein JI: Ductal adenocarcinoma of the prostate diagnosed on needle biopsy: correlation with clinical and radical prostatectomy findings and progression. Am J Surg Pathol 1999;23:1471-1479.

-2 Bostwick DG, Kindrachuk RW, Rouse RV: Prostatic adenocarcinoma with endometrioid features: clinical, pathologic, and ultrastructural findings. Am J Surg Pathol 1985; 9:595-609.
3 Christensen WN, Steinberg G, Walsh PC, Epstein JI: Prostatic duct adenocarcinoma: findings at radical prostatectomy. Cancer 1991;67:2118-2124.

4 Epstein JI, Woodruff JM: Adenocarcinoma of the prostate with endometrioid features: a light microscopic and immunohistochemical study of ten cases. Cancer 1986;57:111119.
Greene LF, Farrow GM, Ravits JM, Tomera FM: Prostatic adenocarcinoma of ductal origin. J Urol 1979;121:303-305.

6 Melicow MM, Tannenbaum M: Endometrial carcinoma of uterus masculinus (prostatic utricle): report of 6 cases. J Urol 1971;106: 892-902.

7 Hameed O, Humphrey PA: Stratified epithelium in prostatic adenocarcinoma: a mimic of high-grade prostatic intraepithelial neoplasia. Mod Pathol 2006;19:899-906. 
$>8$ Tavora F, Epstein JI: High-grade prostatic intraepithelial neoplasia-like ductal adenocarcinoma of the prostate: a clinicopathologic study of 28 cases. Am J Surg Pathol 2008;32: 1060-1067.

$>9$ Herawi M, Epstein JI: Immunohistochemical antibody cocktail staining (p63/HMWCK/AMACR) of ductal adenocarcinoma and Gleason pattern 4 cribriform and noncribriform acinar adenocarcinomas of the prostate. Am J Surg Pathol 2007;31:889-894.

10 Samaratunga H, Singh M: Distribution pattern of basal cells detected by cytokeratin 34 beta E12 in primary prostatic duct adenocarcinoma. Am J Surg Pathol 1997;21:435-440.

11 Owens CL, Epstein JI, Netto GJ: Distinguishing prostatic from colorectal adenocarcinoma on biopsy samples: the role of morphology and immunohistochemistry. Arch Pathol Lab Med 2007;131:599-603.
2 Grignon DJ: Unusual subtypes of prostate cancer. Mod Pathol 2004;17:316-327.

13 Hameed O, Humphrey PA: Immunohistochemistry in diagnostic surgical pathology of the prostate. Semin Diagn Pathol 2005;22: 88-104.

14 Mai KT, Collins JP, Veinot JP: Prostatic adenocarcinoma with urothelial (transitional cell) carcinoma features. Appl Immunohistochem Mol Morphol 2002;10:231-236.

15 Oxley J, Abbott C. Thrombomodulin immunostaining and ductal carcinoma of the prostate. Histopathology 1998;33:391-392.

16 Tu SM, Reyes A, Maa A, Bhowmick D, Pisters LL, Pettaway CA, Lin SH, Troncoso P, Logothetis CJ: Prostate carcinoma with testicular or penile metastases. Clinical, pathologic, and immunohistochemical features. Cancer 2002;94:2610-2617.
Copeland JN, Amin MB, Humphrey PA, Tamboli P, Ro JY, Gal AA: The morphologic spectrum of metastatic prostatic adenocarcinoma to the lung: special emphasis on histologic features overlapping with other pulmonary neoplasms. Am J Clin Pathol 2002;117: 552-557.

18 Ro JY, Ayala AG, Wishnow KI, Ordóñez NG: Prostatic duct adenocarcinoma with endometrioid features: immunohistochemical and electron microscopic study. Semin Diagn Pathol 1988;5:301-311.

19 Epstein JI, Allsbrook WC Jr, Amin MB, Egevad LL, ISUP Grading Committee: The 2005 International Society of Urological Pathology (ISUP) Consensus Conference on Gleason Grading of Prostatic Carcinoma. Am J Surg Pathol 2005;29:1228-1242. 